\title{
Beiträge zur Lehre von der Blutbewegung in den Venen.
}

\author{
II. Mittheilung. \\ Ueber wellenförmige Blutdruckschwankungen im Venensystem. \\ Von
}

Philipp Knoll.

(Hierzu Tafel IX, X, XI.)

Vor drei Jahren machte ich darauf aufmerksam ( ${ }^{1}$, S. 296), dass man beim Kaninchen wellenförmige Schwankungen des Venendruckes zu beobachten vermag, welche Analoga der von mir an den Arterien beobachteten, mit Schauer des Thieres und periodischen Schwankungen der Tiefe und Frequenz der Athmungen einhergehenden Blutdruckschwankungen ${ }^{2}$ ) sind.

Wenige Monate vorher hat $\mathrm{Del}$ e z e nne wellenförmige Schwankungen im Venendruck beschrieben und abgebildet ( ${ }^{3}$, S. 172 u. 176), die er als Traube-Hering'sche bezeichnet - eine Bezeichnung, deren Berechtigung aber weder aus dem Text noch aus den abgebildeten Curven mit Sicherheit zu erschliessen ist. Der erhebliche Wechsel der Pulsgrösse auf den gleichzeitig abgebildeten arteriellen und die stellenweise Ausprägung von Athemdruckschwankungen an den venösen Blutdruckwellen lässt vielmehr weit eher an die von S. Mayer als "Schwankungen durch Interferenz" beschriebenen Wellen $\left({ }^{4}\right.$, S. 22 des S. A.) denken.

So viel ich ermitteln konnte, liegt ausser diesen beiden Mittheilungen über wellenförmige Schwankungen des Venendruckes nur noch die Angabe von R. Klemensiewiez vor (5; S. 116), dass sich an der Curve des venösen Druckes häufig Traube-Hering'sche Wellen erkennen lassen. Da derselbe aber weder nähere Mittheilungen über die Bedingungen macht, unter denen er diese venösen Wellen beobachtete, noch eine genauere Beschreibung oder Abbildung derselben gibt, und an der Stelle, wo er arterielle Traube-Hering'sche Wellen (bei Suspension der künstlichen 
Ventilation) abbildet, sich an der Venencurve, wie er selbst hervorhebt, keine Spur von solchen Wellen findet $\left({ }^{5}, \mathrm{~S}\right.$. 31), so lässt sich kein Urtheil über die Bedeutung seiner Angabe fällen. Es dürfte darum, und mit Rücksicht auf die Bedeutung, welche derartige Druckwellen für die Fragen bezüglich der Innervation der Venen und der Wechselbeziehungen zwischen dem Druck in den Arterien und Venen des grossen Kreislaufs besitzen, wohl gerechtfertigt erscheinen, dass ich in den nachfolgenden Blättern über die Erfahrungen berichte, welche ich hinsichtlich der wellenförmigen Blutdruckschwankungen in der Vena jugularis externa in zahlreichen, an Kaninchen, Katzen und Hunden innerhalb der letzten drei Jahre mittels des von mir beschriebenen Verfahrens zur fortlaufenden Verzeichnung der Druckschwankungen im Venensystem ( ${ }^{6}$, S. 325) ausgeführten Versuchen gemacht habe.

\section{Von der spontanen Athmung abhängige Druckwellen.}

Die durch die einzelnen Athemzüge bedingten Druckschwankungen in der Vena jugularis schmiegen sich, soweit nicht gleichzeitige Veränderungen in der Frequenz des Herzschlages interferiren, wie von vornherein $z u$ erwarten war, den durch die Athmungen erzeugten intrathorakalen Druckschwankungen genau an, verhalten sich also in dieser Richtung wie die, wie ich nachwies, ganz vom Venendruck abhängigen Athemdruckschwankungen der Cerebrospinalflüssigkeit ( 7 , S. 237) und die Athemdruckschwankungen in der Arteria pulmonalis ( $\left.{ }^{8}, \mathrm{~S} .218\right)$. Den respiratorisehen Druckschwankungen in den Arterien eilen sie dementsprechend etwas voraus (Taf. IX Fig. 8, Taf. XI Fig. 3) und bei den seltenen, mit sehr gedehnten activen Exspirationen verknüpften Athmungen, wie sie nach doppelseitiger Vagusdurchschneidung oft vorkommen, können die Fusspunkte der Venendruckwellen mit den Gipfelpunkten der Arteriendruckwellen coïncidiren (Taf. IX Fig. 6)*). Kommt es zu den Eingangs erwähnten periodischen Athmungssehwankungen, so prägen sich analoge wellenförmige Schwankungen im Venendruck aus, bei denen, wie begreiflich, das Sinken im Allgemeinen mit der Beschleunigung, das Steigen mit dem Seltenerwerden der Athmung coinncidirt (Taf. IX Fig. 5 u. 11, Taf. XI Fig. 9), doch können gleichzeitige Veränderungen in der Tiefe der einzelnen Athemzüge, sowie in der Mittellage des Zwerch-

*) Bei der Beurtheilung der fraglichen Curven darf allerdings die Trägheit des an der Arterie verwendeten Quecksilber-Manometers nicht ausser Acht gelassen werden. 
fells bei denselben kleine Abweichungen von dieser Regel bedingen (Taf. IX Fig. 11). Zahl und Länge der gleichzeitig in den Arterien des grossen Kreislaufs sich ausprägenden Druckwellen correspondiren mit jenen in der Vene, doch erscheinen Fuss- und Gipfelpunkte von beiderlei Wellen etwas gegeneinander verschoben (Taf. IX Fig. 11).

Ist die spontane Respiration bei mangelnder oder nur undeutlich an den Athmungscurven ausgeprägter Wellenbildung sehr frequent, so können an der Vene sehr deutliche, unter Umständen sehr verschiedene Länge besitzende Wellen zur Ausprägung kommen (Taf. IX Fig. 2, Taf. X Fig. 2, 2a). Das Grösser- und Kleinerwerden der Pulse am aufsteigenden bezw. absteigenden Theile der venösen und unter Umständen auch die wechselnde Pulsgrösse auf gleichzeitig zur Ausprägung gelangenden arteriellen Wellen (Taf. IX Fig. 2), weisen darauf hin, dass wir es hier mit „Schwankungen durch Interferenz" der sehr frequenten, rhythmisches Sinken und Steigen im Venendruck bedingenden Athemdruckschwankungen mit den durch die Herzthätigkeit bedingten rhythmischen Pulsschwankungen zu thun haben. Die arteriellen und venösen Wellen lassen dabei weder eine Coïncidenz der Fuss- und Gipfelpunkte, noch eine vollständige Uebereinstimmung ihrer Längen erkennen, was im Hinblicke darauf, dass zwischen dem Steigen und Sinken der einzelnen arteriellen und venösen Pulswellen keine Coïncidenz besteht ${ }^{6}$ ), daher also auch die Interferenz derselben mit den einzelnen durch die Athmungen bedingten Druckschwankungen verschieden ausfallen muss, vollständig erklärlich ist.

Die so entstehenden Wellen dureh Interferenz erscheinen aus Gründen, die im nächsten Capitel erörtert werden sollen, an den von der Vene gewonnenen Curven in der Regel weit besser ausgeprägt als an den Arterien, ja es kann vorkommen, dass an der Arterie reflectorisch ausgelöste Blutdruckwellen von ganz anderer Art zum Vorschein kommen, während an der Vene nur. Wellen durch Interferenz verzeichnet werden (Taf. XI Fig. 1).

\section{Von der künstlichen Athmung abhängige Druckwellen.}

Dass die durch einzelne künstliche Athmungen*) bedingten Schwankungen des Venendruckes (Taf. IX Fig. 9, Taf. X Fig. 5 u. 7,

*) Ich bediente mich zur künstlichen Lüftung eines durch einen Motor getriebenen Hering'schen Respirationsapparates für Blasung und Saugung. 
jeweils vor der Marke) sich den durch die künstliche Lüftung erzeugten intrapulmonalen Druckschwankungen genau anschmiegen und dementsprechend den arteriellen respiratorischen Schwankungen gegenüber melar oder weniger stark verschoben erscheinen, bedarf nach dem im vorhergehenden Abschnitt Erörterten kaum einer besonderen Hervorhebung.

Sind die Ventilationen sehr frequent, so kann es zur Bildung von "Wellen durch Interferenz" kommen (Taf. IX Fig. 1, 4, 7, vor der Marke, Taf. XI Fig. 13, vor der Marke, 11, 12). Wenn die einzelnen Ventilationen minder ausgiebig sind, so beherrschen dabei die Pulsschwankungen das Curvenbild, die im aufsteigenden Theil der Welle zu-, im absteigenden abnehmen, und zwar entweder sämmtlich (Taf. IX Fig. 1, wo zumeist Bigemini verzeichnet erscheinen, und Fig. 7) oder wenigstens vorwaltend (Taf. XI Fig. 13). Werden gleichzeitig (bei geöffnetem Thorax) die Zusammenziehungen der Herzabtheilungen nach der von mir angegebenen Methode verzeichnet, so findet man an den betreffenden Curven mit den wellenförmigen Venendruckschwankungen isorhythmische, durch Grösser- und Kleinerwerden der Einzelcurven bedingte Wellen, deren Gipfel- und Fusspunkte aber am Vorhof und Ventrikel nicht coincidiren, während die Vergrösserung und Verkleinerung der vom rechten Vorhof gewonnenen Curven parallel geht mit der $\mathrm{Zu}$ - und Abnahme der Venenpulscurven (Taf. IX Fig. 7).

Ich vermag nicht mit voller Sicherheit anzugeben, welcher Factor unter diesen Umständen für die $A b$ - und Zunahme der einzelnen, von den Herzabtheilungen gewonnenen Curven maassgebend ist. Jede einzelne Einblasung hemmt nämlich die Diastole und bedingt dadurch eine Verkürzung des absteigenden Curvenschenkels, sie drängt aber zugleich das Herz gegen die Thoraxöffnung, was an und für sich wieder eine Verlängerung dieses Curvenschenkels zur Folge haben muss. Andrerseits aber bedingt wieder die einzelne Aussaugung eine Begünstigung der Diastole und ein Zurücksinken des Herzens gegen die Wirbelsäule mit den vorigen gegenüber umgekehrten Wirkungen auf den diastolischen Curvenschenkel. Bei dem systolischen Curvenschenkel dagegen dürfte nur die Verlängerung beim Zurücksinken und die Verkürzung beim Gehobenwerden des Herzens in Betracht kommen. Es spielen also mit den Zusammenziehungen des Herzens nicht zusammenhängende mechanische Momente eine Rolle bei dem Zustandekommen der dureh $\mathrm{Ab}$ - und Zunahme der 
einzelnen von den Herzabtheilungen gewonnenen Curven bedingten Wellen. Doch weist der. Umstand, dass die Curvenzu- und -abnahme in der Vene parallel geht mit der hauptsächlich durch Verlängerung und Verkürzung des diastolischen Schenkels bedingten $\mathrm{Zu}$ - und $\mathrm{Ab}$ nahme der Vorhofscurven immerhin darauf, dass die Begünstigung und Hemmung der Diastole und folgeweise die grössere oder geringere Ausgiebigkeit der darauf folgenden Systolen das ausschlaggebende Moment für die $\mathrm{Ab}-$ und Zunahme der vom Vorhof und Ventrikel gewonnenen Curven ist. Wir müssen danach also annehmen, dass. während der Curvenzunahme die Saugungen und während deren Abnahme die Blasungen mit den einzelnen Diastolen zusammenfallen. Dass. aber Fuss- und Gipfelpunkte der so entstehenden Wellen am Vorhof und Ventrikel mit einander nicht cö̈ncidiren, erklärt sich zur Genüge aus dem Umstande, dass ja auch die Diastolen an Vorhof und Ventrikel nicht gleichzeitig eintreten. Aus den eben gemachten Auseinandersetzungen geht aber hervor, dass bei den "Schwankungen durch Interferenz" nicht blos die Einwirkung der Blasungen und Saugungen auf die Blutgefässe, sondern auch, und vielleicht sogar in erster Reihe, jene auf das Herz in Betracht kommt. Sind die einzelnen, $z \mathfrak{u}$ "Schwankungen durch Interferenz" führenden, sehr frequenten, künstlichen Ventilationen ausgiebig; so beherrschen die durch letztere bedingten respiratorischen Druckschwankungen das Curvenbild an der Vene (Taf. IX Fig. 4, Taf. XI Fig. 11, 12, wo, wie der Vergleich mit der darunter stehenden arteriellen Druckcurve lehrt, immer 2-3 Pulse auf eine der grösseren Druckschwankungen an den Venencurven kommen).

Auf- und Absteigen der Curvenreihe an den venösen Wellen wird dabei entweder hauptsächlich durch Länger- und Kürzerwerden des aufsteigenden (Taf. XI Fig. 12) oder vorwaltend durch Kürzerund Längerwerden des absteigenden Schenkels der respiratorischen Druckwellen bedingt (Taf. IX Fig. 4, Taf. XI Fig. 11). Im ersten Falle summiren sich also die Wirkungen der Venenpulse mit jenen der Blasungen, im letzteren Falle aber vermindern sie die Wirkung der Aussaugungen.

An den gleichzeitig verzeichneten arteriellen Druckeurven sind die "Schwankungen durch Interferenz" oft viel weniger ausgeprägt als an der Vene (Taf. IX Fig. 1, vor der Marke, Taf. X Fig. 2, 2a, Taf. XI Fig. 12), oder sie fehlen ganz (Taf. XI Fig. 13), oder es erscheinen an ihrer Stelle "spontane Blutdruckschwankungen" 
(S. Mayer) ausgeprägt (Taf. XI Fig. 11). Das Vorhandensein solcher spontaner arterieller Blutdruckschwankungen kann übrigens während frequenter künstlicher Ventilation durch das Auftreten von "Wellen durch Interferenz" bis zu einem gewissen Grade verdeckt werden und erst mit dem Aussetzen der künstlichen Ventilation klar zu Tage treten (Taf. IX Fig. 1).

Dass in den Venen bei dem niedrigen Druck, der in denselben herrscht, sowie in Folge der relativen Dünne der Wandungen des rechten Herzens gegenüber dem linken Ventrikel der mechanische Effect der Blasungen und Saugungen sich stärker ausprägt als in den Arterien, ist wohl begreiflich. Beim Vergleich der beiden Curven muss aber auch die geringere Empfindlichkeit der an den Arterien verwendeten graphischen Apparate in Betracht gezogen werden, von denen namentlich das Hürthle'sche Kautschukmanometer, wenn die Membran etwas dicker ist, wellenförmige Blutdruckschwankungen, nur wenn sie recht erheblich sind, wiedergibt. Ferner ist darauf hinzuweisen, dass eine Gerinnselbildung im Blutgefässe oder in der in dasselbe eingeführten Canüle unter Umständen wohl nicht die Ausprägung der Pulse und jäherer, stärkerer Blutdrucksteigerungen, wohl aber jene von flacheren, wellenförmigen Druckschwankungen hintanzuhalten vermag.

\section{Druckwellen bei Dyspnoe.}

Ich wählte diese Ueberschrift für die nachfolgenden Mittheilungen, weil nicht alle Erscheinungen, über die ich in denselben zu berichten habe, in das für die Traube-Hering'schen Wellen aufgestellte Schema sich einfügen, auch die Entstehungsursache der Druckwellen im Venensystem bei Dyspnoe eine andere ist als jene der arteriellen Druckwellen.

Wohl kann man bei curarisirten Hunden, Katzen und Kaninchen beim Aussetzen der künstlichen Ventilation im Venensystem den Traube-Hering'schen ähnelnde Druckwellen oft beobachten.' Bei näherer Beobachtung ergibt sich aber, dass diese Wellen in einer Anzahl von Fällen durch das Auftreten sehr seltener, tieferer oder seichterer Respirationen bedingt sind, die ein bald stärker, bald schwächer ausgeprägtes Sinken des Venendruckes bei der Ein- und ein Steigen desselben bei der Ausathmung bedingén, während gleichzeitig auch an der Arterie bald mehr, bald minder beträchtliche oder bei Verwendung eines weniger empfindlichen graphischen Apparates wohl auch gar keine Druck- 
wellen zur Ausprägung kommen (Taf. IX Fig. 3 u. 4, nach der Marke). Verzeichnet man gleichzeitig die Volumschwankungen des Herzens vom Perikard aus ${ }^{9}$ ), so finden sich auch an diesen Curven Wellen, die durch Hebung und Senkung der Curvenreihe und Grösser- und Kleinerwerden der Einzelcurven bedingt werden (Taf. IX Fig. 3), und zwar werden die Curven während der Exspiration, welche die Blutzufuhr zum Herzen hemmt, und damit auch die Volumschwankungen desselben herabsetzt, kleiner, und während der Inspiration aus entgegengesetzten Gründen grösser. Dazu kommt ausserdem, dass jede Einathmung in Folge der Ausdehnung der Lunge zu einem Druck auf das zwischen den Lungen wie in einer Nische befindliche lufthaltige Perikard, und damit zu einer Druckerhöhung in dem mit demselben verbundenen graphischen System führt, während der exspiratorische Lungencollaps die entgegengesetzte Wirkung ausübt.

Die Seltenheit dieser, bei unvollständig curarisirten, vagotomirten und nicht vagotomirten Thieren beim Aussetzen der künstlichen Lüftung oder, wenn diese ungenügend ist, schon während derselben auftretenden spontanen Athmungen, der anfangs nicht selten rein inspiratorische Charakter derselben und die längeren Pausen zwischen denselben erinnerten mich an den bei Wiederbelebung der Athmung nach Erstickung auftretenden Athmungstypus $\left.{ }^{10}\right)$, und legten mir den Gedanken nahe, dass das Curare von dem schon Nikolski und Dogiel (11, S. 96) öhne genauere Begründung behaupteten, dass es auf das Athemcentrum selbst und nicht bloss auf die Athmungsmuskelnerven wirke, möglicher Weise Veränderungen im Athemeentrum bedinge, welche die erwähnte Art der Athmung nach sich ziehen. Einige vorläufig von mir in dieser Richtung angestellte Versuche, in denen bei vorsichtig abgestufter Curarisirung vor eintretender vollständiger Paralyse sehr seltene, durch Pausen getrennte Respirationen und Druckwellen in der Arterie und Vene zu beobachten waren (Taf. X Fig. 6,8), sprechen hierfür. Doch möchte ich ein abschliessendes Urtheil nicht abgeben, ehe die Frage ar: einer grösseren Zahl von Thieren unter verschiedenen Versuchsbedingungen und mit verschiedenen Curare-Sorten untersucht wurde.

Für die Frage nach der Entstehung der Traube-Her ing'schen Wellen ist diese Sache ersichtlich von Wichtigkeit. Denn dürfen wir annehmen, dass die Seltenheit der Athmung unter diesen Umständen nicht etwa dadurch bedingt wird, dass mehrere Impulse vom Athmungscentrum ausgesendet werden müssen, um die paretischen 
Athmungsmuskelnerven zu erregen, sondern dass das durch das Curare veränderte Athemcentrum diese Impulse ungewöhnlich selten entsendet, so spricht dies sehr zu Gunsten der von Cyon. $\left({ }^{12}\right.$, S. 273,274$)$ bestrittenen Ansicht Hering's ( ${ }^{13}$, S. 14, des S. A.), dass diese Wellen „durch eine periodische, der Innervation der quergestreiften Athmungsmuskeln associirte Innervation der Gefässnerven, welche nicht durch das Curare gelähmt sind, entstehen". Es würde dann der gegen diese Ansicht erhobene Finwand entfallen, dass der Rhythmus der Traube-Hering'schen Wellen weder dem normalen, noch dem nach Vagusdurchschneidung zu beobachtenden Athmungsrhythmus entspricht, und damit auch, wenigstens für diese Art von Blutdruckwellen die Hülfshypothese S. M a y er's unnöthig werden, dass "zwischen das Athemcentrum und den peripheren gefässbewegenden Apparat ein Centrum eingeschaltet ist, in welchem bei normaler Action des Athemcentrums sich erst mehrere von dort kommende Innervationen summiren müssen, um gleichsam eine Entladung des Centrums für die Gefässinnervation hervorzurufen" $\left({ }^{4}\right.$, S. 17 des S. A.).

Dass die fragliche Art der Athmung bei unvollständig curarisirten Thieren nicht ausreicht, das Blut eupnoisch zu erhalten, ergibt sich unter Anderem aus der nach dem Aussetzen der künstlichen Lüftung auftretenden allmäligen Abnahme der Volumschwankungen' des Herzens, der damit zusammenhängenden Zunahme des venösen, sowie aus der Steigerung des arteriellen Druckes (Taf. IX Fig. 3, Taf. X Fig. 6, 8).

In einer anderen Reihe von Fällen treten beim Aussetzen der künstlichen Lüftung keine wirklichen Athembewegungen auf, sondern uur schwache Zuckungen in exspiratorisch wirkenden Muskeln, namentlich den Bauchmuskeln, die zu jähen, rasch vorübergehenden Drucksteigerungen in der Vene führen, während an der arteriellen Druckcurve oft kaum eine Veränderung zu erkennen ist ('Taf. XI Fig. 8).

In einer weiteren Reihe von Fällen treten mit dem Aussetzen der künstlichen Lüftung in der Vene und in der Arterie Wellen auf, die im Wesentlichen durch periodisches Häufiger- und Seltenerwerden der Pulse bedingt werden und, weil die Pulszunahme, wie ich in einer späteren Mittheilung noch eingehender darlegen werde, in der Vene eine Drucksenkung bewirkt, in der Arterie aber eine Drucksteigerung, einen entgegengesetzten Verlauf zeigen (Taf. X 
Fig. 5). Solehe durch Wechsel der Pulsfrequenz bedingte, in Vene und Arterie entgegengesetzt gerichtete Wellen, die ich nur bei erhöhtem Tonus der Herzhemmungsfasern beobachtet habe, können unter den letzteren Umständen auch während der künstlichen Lüftung auftreten, wenn dieselbe entweder an and für sich, oder etwa wegen bestehenden Lungenödems insufficient ist (Taf. X Fig. 7).

Die periodische Beschleunigung des Pulses coinncidirte in diesen Fällen wohl mit leichten Zuckungen, die bei demselben Thiere, wenn der Vagustonus nicht erhöht war, nur zu den vorher beschriebenen flüchtigen Drucksteigerungen in der Vene führten, Inspirationen aber, die zu reflectorischer Herabsetzung des Vagustonus hätten führen könnèn, waren nicht zu bemerken. Ich konnte sie übrigens auch bei sehr frequenter spontaner Athmung in Verbindung mit leichtem Schauer des Thieres bei einem Kaninchen beobachten, bei welchem reflectorisch, durch mechanische Reizung der Trigeminusendigungen in der Nase eine sehr lang anhaltende Erhöhung des Vagustonus ausgelöst worden war (Taf. XI Fig. 5). Da ich oft die Beobachtung machte, dass sensible Reize, und zwar selbst solche, welche sonst regelmässig ein Seltenerwerden des Herzschlages herbeiführen, eine Pulsbeschleunigung hervorrufen, wenn sie während bestehender Erhöhung des Vagustonus einwirken, glaube ich diese durch periodischen Wechsel der Frequenz des Herzschlages bedingten Druckwellen nach Analogie der periodischen Athmungs- und Blutdruckschwankungen (2, S. 446) durch eine dauernde Reizung sensibler Nerven, durch .das dyspnoische Blut, die nur zu einer zeitweisen Entladung im Vaguscentrum führt, erklären zu sollen. Der Gedanke, dass auch die vorher erwähnten periodisch auftretenden Zuckungen von Exspirationsmuskeln, durch eine solche sensible Reizung ausgelöst werden, liegt nicht fern.

In einer vierten Reihe von Fällen beobachtete ich beim Aussetzen der künstlichen Lüftung bei curarisirten Katzen und Hunden, die nur schwache Zuckungen erkennen liessen, neben relativ hohen Traube-Hering'schen Wellen in der Arterie wohl ausgeprägte Wellen von annähernd gleicher Länge in der Vene (Taf. X, Fig. 1, 4). Nähere Betrachtung der Curven lehrte, dass in diesen Fällen bei den venösen Wellen die Drucksteigerung mit einer Abnahme und die Senkung mit einer Zunahme der Grösse der einzelnen Pulse zusammenfiel. Dabei machte sich unter Umständen eine fortschreitende Absehwächung der Contractionen des rechten Vorhofes 
unter der Einwirkung des dyspnoischen Blutes (14, S. 597) durch eine allmälige Abnahme der Grösse der Venenpulse und eine damit Hand in Hand gehende Steigerung des Venendruckes im Ganzen bemerkbar (Taf. X Fig. 1, 4).

Diese im Curvenbilde den Traube-Hering' schen Wellen so ähnelnden venösen Druckschwankungen lassen in ihrem Verlauf im Grossen und Ganzen einen gewissen Parallelismus mit den gleichzeitig verzeichneten arteriellen Wellen erkennen, wenn auch die Fuss- und Gipfelpunkte beider in der Regel nicht coïncidiren. Dagegen aber, dass sie gleich den arteriellen Wellen durch eine der Erregung der arteriellen Vasomotoren associirte, periodische Thätigkeit von vasomotorischen, an die Venen herantretenden Nerven bedingt sind oder durch wechselnde Blutzufuhr zum rechten Herzen in Folge des Wechsels des Lumens der Arterien, spricht sehon der Umstand, dass diese Wellen im Venensystem beim Aussetzen der künstlichen Ventilation, bei Thieren, bei denen sie ursprünglich mit Traube-Hering'schen Wellen verknüpft auftraten, auch dann zu finden sind, wenn in Folge mehrmals erzeugter Dyspnoe oder stärkerer Curarisirung die Drucksteigerung und Wellenbildung in der Arterie ganz ausbleibt (Taf. XI, Fig. 6). Auch lässt sich zuweilen beobachten, dass die Wellenbildung in der Vene erst dann recht deutlich hervortritt, wenn der arterielle Druck sinkt und kaum noch Andeutungen von wellenförmigen Schwankuugen desselben $\mathrm{zu}$ finden sind (Taf. X Fig. 3).

Nähere Beobachtung der betreffenden Versuchsthiere ergibt, dass diese venösen Wellen mit bald jäher, bald träger ablaufenden $\mathrm{Zu}-$ sammenziehungen und Erschlaffungen der Halsmuskeln, namentlich der Sternocleidomastoidei coïncidiren, welche zu einer Zerrung an dem frei präparirten Theil der Vena jugularis externa vor der vorderen Brustapertur und damit zu einer periodischen Verengerung des Lumens der Vene, Pulsverkleinerung und Drucksteigerung führen.

Man kann denn auch durch einen ganz leichten Zug an dem Sternocleidomastoideus ganz analoge Wellen erzeugen, und besonders schlagend ist das Ergebniss dieses Experimentes hinsichtlich der Entstehung der fraglichen Wellen, wenn man dasselbe unmittelbar nach dem Aussetzen der künstlichen Ventilation (Taf. IX Fig. 10, bei a), wo noch keine solchen Contractionen der Halsmuskeln auftreten, in Fällen ausführt, in denen später derartige Contractionen und Wellen spontan sich einstellen (ebenda bei $b$ ). 
In geeigneten Fällen lässt sich feststellen, dass die oben erwähnten Zusammenziehungen der Halsmuskeln mit flachen Athembewegungen zusammenfallen, dass wir also in denselben, wie von vornherein zu vermuthen war, nur den Ausdruck von Athmungsinnervationen zu erblicken haben. Unter Umständen nun combiniren sich sehr seltene, derartige, mit Halsmuskelcontractionen einhergehende Athmungen mit häufiger auftretenden, jähen Muskelzuckungen (Taf. XI Fig. 7 bei a), wodurch ein eigenthümlich zusammengesetztes Wellensystem in Arterien und Venen zu Stande kommt.

Druckwellen in der Vene beim Aussetzen der künstlichen Ventilation an curarisirten Thieren können endlich auch durch periodischen Wechsel in der Energie der Thätigkeit des Herzens im Ganzen oder wenigstens des rechten Vorhofes erzeugt werden. Im ersteren Falle kann man einen analogen Wechsel der Pulsgrösse in der Arterie und Vene bei entgegengesetztem. Verlauf der Druckwellen beobachten (Taf. XI Fig. 10). Durch vorsichtige Vergiftung mit Helleboreïn, die, wie ich nachwies, periodische Abschwächung und Verstärkung der Herzthätigkeit erzeugt (15, S. 47), lassen sich ganz analoge, in den beiden Gefässarten entgegengesetzt gerichtete Wellen hervorrufen (Taf. X Fig. 9). - Von den in die letztere Gruppe gehörenden Fällen scheint mir der, im Curvenbilde den von F an o entdeckten, den Vorhofstonusschwankungen bei Kaltblütern $\left({ }^{16}, \mathrm{~S} .4-8\right)$ gleichende, zu flachen Druckwellen in der Vene führende, periodische Wechsel in den Zusammenziehungen des rechten Vorhofes, den ich wiederholt bei Katzen beobachtete, besonders bemerkenswerth (Taf, XI, Fig. 4).

\section{Schlussbemerkungen.}

Aus den vorstehenden Mittheilungen erhellt, dass wellenförmige Blutdruckschwankungen in der Vena jugularis, in der die in der Vena cava superior und im rechten Vorhof sich vollziehenden Druckschwankungen verzeichnet werden, kaum minder häufig zur Beobachtung gelangen als im Arteriensystem. Ja, die durch Interferenz der Puls- und Athemdruckschwankungen entstehenden „Wellen durch Interferenz" finden sich, bedingt dureh den relativ stärkeren Einfluss der Athemdrucksehwankungen auf das rechte Herz und das Venensystem, hier häufiger und ausgeprägter als in den Arterien. Auch von periodischen Athmungsschwankungen abhängige Wellen im 
Venensystem, für die ich schon in einer früheren Mittheilung unter der nach S. Mayer's Definition nicht ganz zutreffenden Bezeichnung "Spontane Blutdruckschwankungen" ein paar Beispiele gegeben $(1$, S. 294, 295), finden sich häufig. Dagegen fehlen in der Vene die durch periodischen Wechsel im Lumen der Gefässe bedingten eigentlichen "spontanen Blutdruckschwankungen" (S. M a y e r) und die Traube-Hering'schen Wellen, neben welchen letzteren aber unter Umständen analog verlaufende, aber durch Wechsel in der Pulsgrösse besonders charakterisirte Wellen im Venensystem sich finden, die mechanisch durch von der periodisch sich vollziehenden Athmungsinnervation ausgelöste Contractionen der Halsmuskeln bedingt werden. Die bei ausreichend künstlich ventilirtèn, curarisirten Thieren $\mathrm{zu}$ beobachtenden "spontanen Blutdruckschwankungen" im Arteriensysteme habe ich in den letzten Jahren, wohl im Zusammenhang mit der so manchen Kymographionversuch erschwerenden, veränderten Beschaffenheit des Curare, überbaupt nicht häufig und dann in der Regel nur von geringerer Höhe gesehen. Wenn neben ihnen Wellen im Venensystem erschienen, so waren es immer solche "durch Interferenz". So bieten also die im Venensystem auftretenden Druckwellen keine Anhaltspunkte für die Annahme einer gleichzeitigen Erregung von Vasomotoren des Venensystems mit jenen der Arterien.

Es wird Aufgabe einer nächsten Mittheilung sein, darzulegen, ob sich überhaupt Anzeichen dafür auffinden lassen, dass Reize, welche zu einer Erregung der arteriellen Vasomotoren führen, auch eine solche von Vasomotoren des Venensystems hervorrufen.

\section{Literatur-Verzeichniss.}

1) $\mathrm{Ph}$. Knoll, Bemerkungen zur Infusion blutwarmer physiologischer Kochsalzlösung in das Gefässsystem: Arch. f. experim. Pathologie u. Pharmakologie Bd. 36 S. $293 \mathrm{ff}$.

2) Derselbe, Ueber periodische Athmungs- und Blutdruckschwankungen. Sitzungsber. d. k. Akad. d. Wissensch. Bd. 92 Abth. 3 S. 439 ff. 1885.

3) M. E. Delezenne, Sur les variations de la pression veineuse. Arch. de physiol: norm. et pathol. p. $170 \mathrm{ff}$. 1895 .

4) S. Mayer, Studien zur Physiologie des Herzens und der Blutgefässe. Fünfte Abhandlung. Ueber spontane Blutdruckschwankungen. Sitzungsber. d. k. Akad. d. Wissensch. Bd. 74 Abth. 3. 1876. 
5) R. Klemensiewicz, Experimentelle Beiträge zur Kenntniss des normalen und pathologischen Blutstromes. Ebenda Bd. 94. 1886.

6) Ph. Knoll, Beiträge zur Lehre von der Blutbewegung in den Venen. Erste Mittheilung. Ueber den Venenpuls. Pflüger's Archiv Bd. $72 \mathrm{~S} .317 \mathrm{ff}$.

7) Derselbe, Ueber die Druckschwankungen in der Cerebrospinalflüssigkeit und den Wechsel in der Blutfülle des centralen Nervensystems. Sitzungsber. d. k. Akad, d. Wissensch. Bd. 93 Abth. 3 S. 227 ff. 1886.

8) Derselbe, Der Blutdruck in der Arteria pulmonalis bei Kaninchen und seine respiratorischen Schwankungen. Ebenda Bd. 97 Abth. 3 S. 207 ff. 1888.

9). Derselbe, Ueber eine Methode zur Verzeichnung der Volumschwankungen des Herzens. Ebenda Bd. 82 Abth. 3 S. 7 ff. 1880.

10) M. Pick und Ph. Knoll, Ueber die Erscheinungen bei Wiederbelebung der durch Erstickung oder Chloroformzufuhr vernichteten Athmung. Arch. f. experim. Pathol. u. Pharmak. Bd. 40 S. $81 \mathrm{ff}$.

11) Wl. Nikolski u. Joh. Dogiel, Zur Lehre über die physiologische Wirkung des Curare. Pflüger's Archiv Bd. 47 S. $68 \mathrm{ff}$.

12) E. von Cyon, Beiträge zur Physiologie der Schilddrüse und des Herzens. Pflüg er's Archiv Bd. 70 S. 126 ff.

13) E. Hering, Ueber den Einfluss der Athmung auf den Kreislauf. Erste Mittheilung. Ueber Athembewegungen des Gefässsystems. Sitzungsber. d. k. Akad. d. Wissensch. Bd. 60 Abth. 2. 1869.

14) Ph. Kn oll, Ueber die Wirkungen des Herzvagus bei Warmblütern. P flüger's Arch. Bd. 67 S. $587 \mathrm{ff}$.

15) Derselbe, Ueber Incongruenz in der Thätigkeit der beiden Herzhälften. Sitzungsber. d. k. Akad. d. Wissensch. Bd. 99 Abth. 3 S. 32 ff. 1890.

16) Ph. Bottazzi, The oscillations of the auricular tonus in the batrachian heart with a theory on the function of sarcoplasma in muscular tissues. Journal of physiology vol. 21 p. 1 ff. 1897.

\section{Erklärung der Abbildungen auf Tafel IX-XI.}

Sämmtliche Figuren sind von links nach rechts zu lesen. Die niederen Striche auf der Abscisse markiren Secunden, die höheren, durch eine zweite Horizontale verbundenen den Zeitpunkt und eventuell die Dauer eines Eingriffes. Die mit $J$. bezeichneten Curvenreihen geben die Druckschwankungen in der Vena jugularis externa, die mit $C$. bezeichneten jene in der Karotis wieder, erstere mit dem in einer früheren Abhandlung geschilderten Verfahren, letztere bei Fig. 4 Taf. IX mit einem Hürthle'schen Kautschuk-, sonst aber mit einem Quecksilbermanometer verzeichnet. $R$. sind Athmuugscurven, gewonnen durch Verbindung der Trachealcanüle des Versuchsthieres mit einem geschlossenen, mit einer Marey'schen Scbreibtrommel communicirenden Luftraum. Die aufsteigende Linie zeigt die Exspiration an.

Die mit $A$. bezeichneten Curven geben, in der aufsteigenden Linie, die Zusammenziehungen des rechten Vorhofes, die mit $V$. bezeichneten jene des Ventrikels 
634 Philipp Knoll: Beiträge zur Lehre von der Blutbewegung in den Venen.

wieder. $P$. verzeichnet die Volumschwankungen des Herzens durch Verbindung des lufthaltigen, von einer Caniile durchbohrten Perikard mit einer Schreibtrommel.

Fig. 9 anf Taf. IX und 5-8 anf Taf. $X$ stammen von narkotisirten und curarisirten Hunden, Fig. 10 auf Taf. IX, 1, 3, 4 auf Taf. X, sowie 4, 6, 7 und 10 auf Taf. XI von narkotisirten und curarisirten Katzen her. Fig. 2, 5, 6, 8 und 11 auf Taf. IX; 2 und 2 a auf Taf. $X$, sowie $1-3,5$ und 9 auf Taf. XI stammen von unvergifteten, 1, 3, 4 und 7 auf Taf. IX dagegen und 9 auf Taf. $X$, sowie 8 und 11-13 auf Taf. XI von curarisirten Kaninchen her. Fig. 2, 2a auf Taf. X und 1-3, sowie 5 auf Taf. XI wurden von demselben Kaninchen gewonnen, und zwar Fig. 2 auf Taf. XI zu Beginn und 3 am Schluss des Versuches, nach Section beider Halsvagi, die übrigen Curven in der Zwischenzeit.

Bei Fig. 1 auf Taf. XI zeigt die Marke mechanische Reizung der Trigeminusendigungen in der Nase an. Im Uebrigen bedeuten die Marken und die erste Marke auf Fig. 7 Taf. X Aussetzen der künstlichen Respiration, die zweite Marke auf der angegehenen Figur aber Wiederaufnahme der Ventilation.

Fig. 5 auf Taf. XI wurde während einer anhaltenden, durch mechanische Reizung der Trigeminus-Endigungen in der Nase erzeugten Erregung der Hemmungsfasern des Herzens gewonnen, Fig. 10 auf Taf. IX, sowie 1, 3, 4, 6, 8 auf Taf. $\mathrm{X}$ und 7, 8 und 10 auf Taf. XI während des Aussetzens der künstlichen Ventilation. Das Thier, von welchem Fig. 9 auf Taf. X gewonnen wurde, war mit 0,01 Helleboreïn vergiftet. 


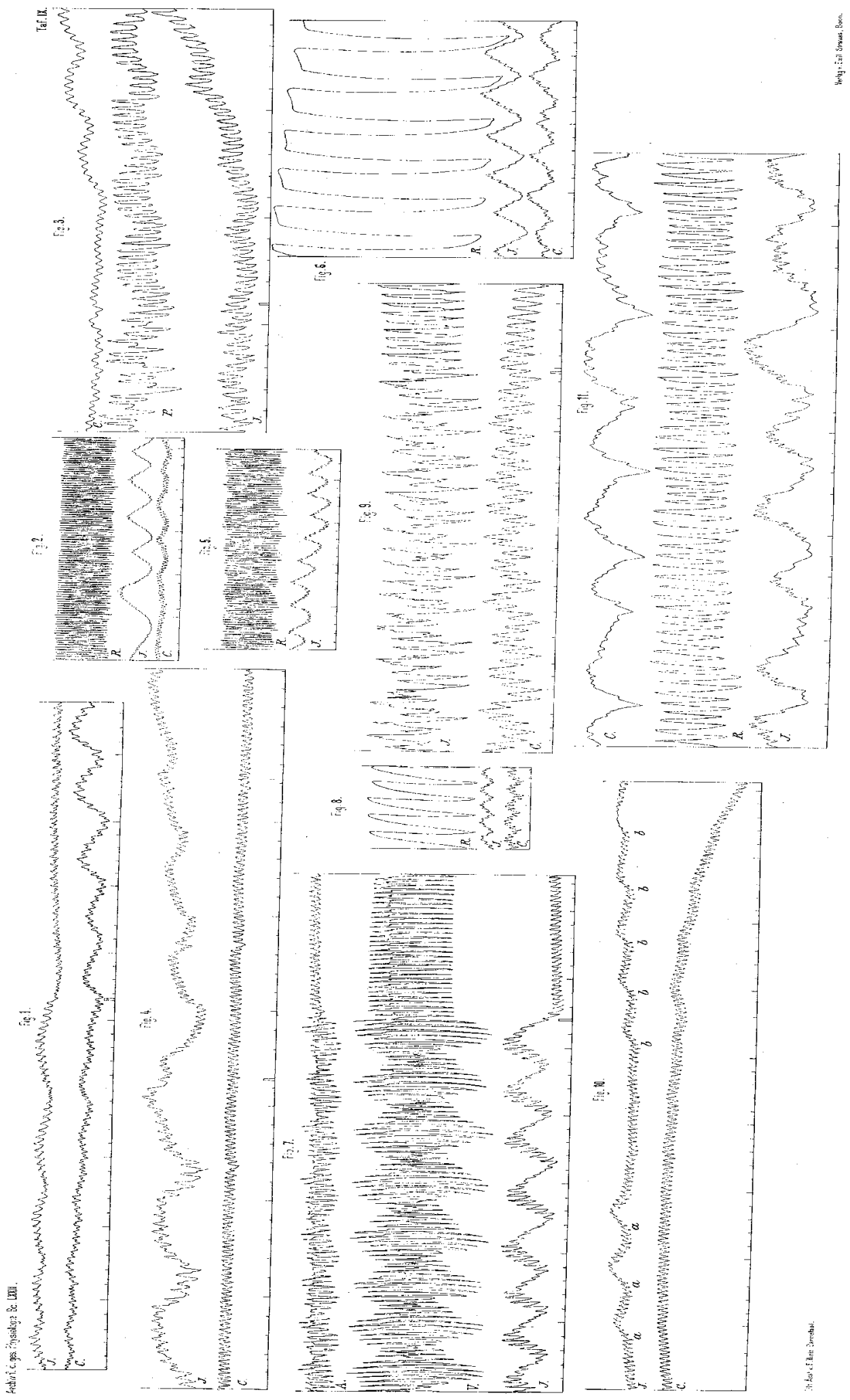




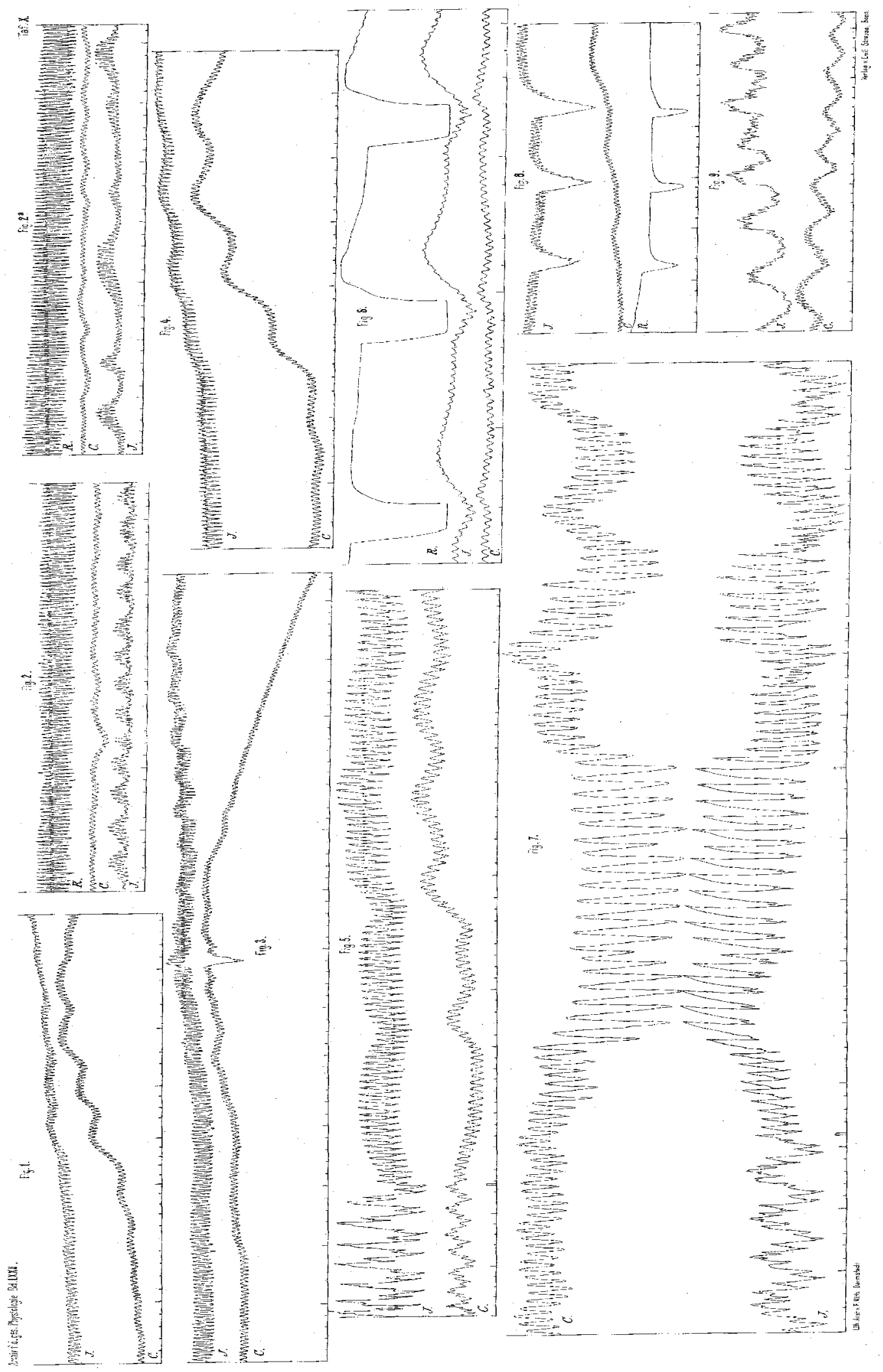




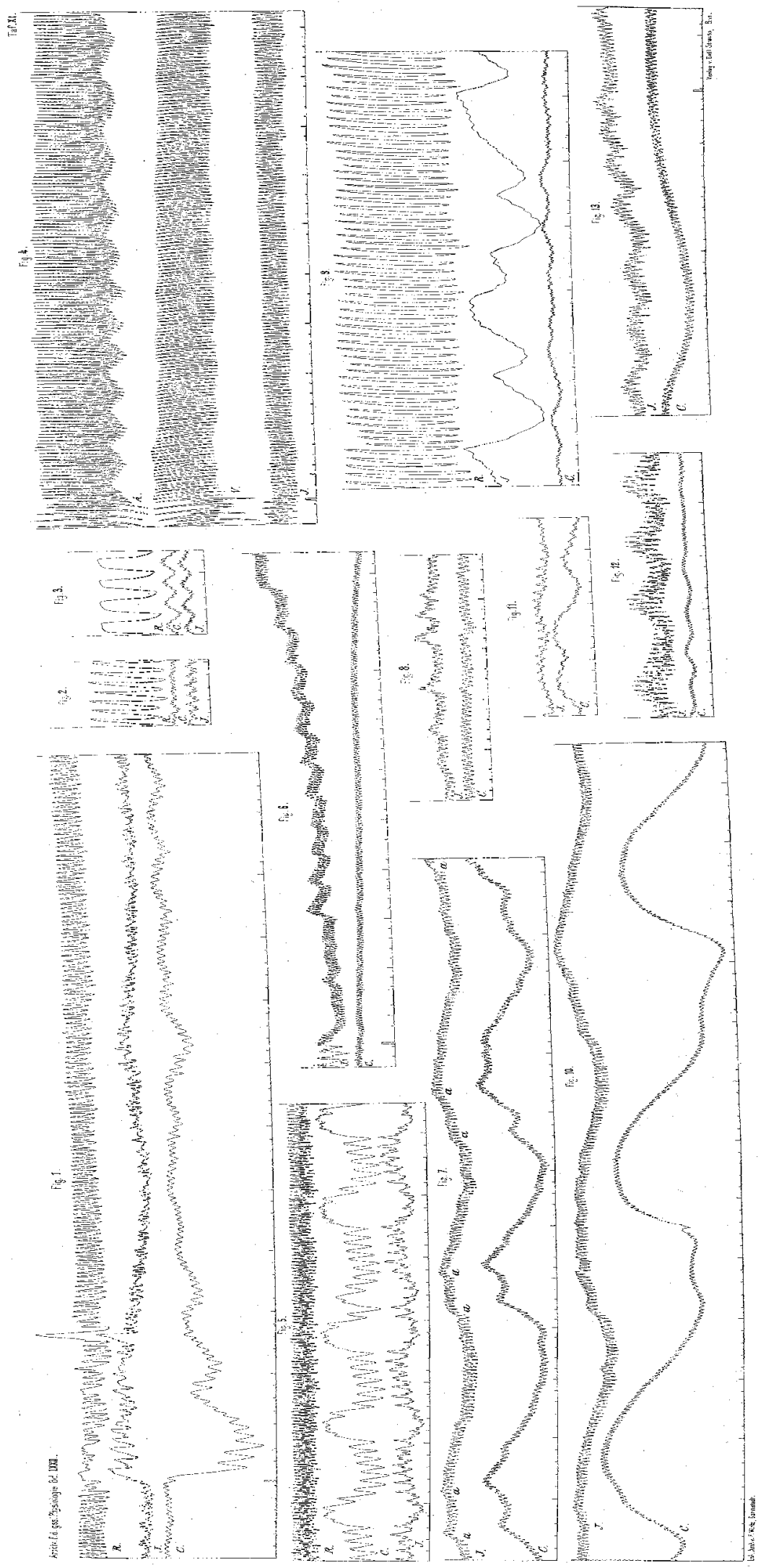

